\title{
The neutral assumption and maintenance of colour morph frequency in mangrove snails
}

\author{
L. M. COOK \\ Department of Environmental Biology, University of Manchester, Manchester M13 9PL, U.K.
}

\begin{abstract}
The leaf-living mangrove snail Littoraria pallescens is polymorphic for shell colour, and morph frequencies vary little throughout the species range. Constancy may be due to stabilizing selection or large effective population size. Using variances of morph frequency obtained from sample data, estimates of $4 N_{\mathrm{e}}(v+w)$ have been made $\left(N_{\mathrm{e}}\right.$ is effective population size and $v$ and $w$ mutation rates for two shell colour morphs). On the assumption of neutrality, the estimates are 12.2 or 25.2, depending on dominance, suggesting that $N_{\mathrm{e}}$ is probably of the order of $10^{6}$ or more. Stabilizing selection could, however, have inflated these values.

If a set of estimates from isozyme data indicated a much lower effective population size, then the supposition of stabilizing selection would be reinforced. Conversely, if they indicate a similar size there would be no reason to reject the neutral hypothesis. However, apparent mutation rate depends on detectability of phenotypic differences, and may vary by orders of magnitude between classes of data. The relation of apparent phenotypic conversion to base mutation is outlined. Instead of looking at frequency distributions of multiple alleles at allozyme loci, or the number of alleles present, the alleles may be pooled to give the same number of morphs as the colour locus. This avoids the problem of comparability of mutation rates. Published allozyme data from a congeneric species give estimates of effective population size similar to those for $L$. pallescens morphs.
\end{abstract}

Keywords: effective population size, Littoraria pallescens, neutral mutation, mangrove snail.

\section{Introduction}

A difference in shell colour and pattern between individuals in mollusc populations is one of the important classes of genetic polymorphism. The most thoroughly worked example is Cepaea nemoralis. Despite 50 years of intensive research, the reasons for the polymorphism are still not agreed or understood (Cain, 1983; Clarke, et al., 1978; Jones et al., 1977). The most likely causes are selection by predators, coupled with physiological differences between morphs. These establish a balance (a) through differential selection in different parts of a heterogeneous environment, opposed by the homogenizing effect of migration, or (b) through the effect of apostatic selection, or both. It has been argued, however, that the effect of selection is overemphasized, and that much of the variability arises from mutation of usually more or less equivalent alleles, founder effect and drift (Selander \& Ochman, 1983). The question how shell colour polymorphisms are generally maintained deserves to be settled.

One reason why these factors are difficult to distinguish in $C$. nemoralis is that it is long-lived and occupies a wide variety of habitats, which have changed through climatic effects and human interference over a time scale which is relatively short compared with the time scale of gene frequency changes in the snail. This makes it difficult to detect, or rule out, critical associations between morph frequency and habitat (Clarke et al., 1978; Cameron \& Dillon, 1984, Ochman et al., 1983).

In the Indo-Pacific region, the genus Littoraria includes polymorphic species for which these limitations do not apply (Reid, 1986). Some of the species are effectively restricted to bark surfaces. Although there is variation in shell colour, the colours tend to match the background, so that bark species are more or less cryptic. Others live almost entirely on leaves. In this group there are several species, sometimes very abundant, which tend to be polymorphic for colour morphs, usually yellow, orange and a dark olive colour. The genetics are unknown, but work on other molluscs (Murray, 1975) suggests, by analogy, that this variation is genetic in origin, and can probably be resolved into a locus which controls yellow versus orange (and possibly unpigmented) ground colour, and another 
with alleles for the presence or absence of dark pigment. The latter is analagous to the banding loci of other molluscs. The habitat of these species is relatively undisturbed and is very widespread. Two of the favoured tree genera, Rhizophora and Avicennia, each have species with similar foliage living throughout the tropics, so that the snails occupy the same habitats wherever they are found. Conditions on the two types of tree may sometimes be compared at the same site. The difference in the appearance of bark-living and leaf-living species in the same genus, and the constancy of their habitat give these snails substantial advantages over Cepaea species as a model system to study molluscan shell colour polymorphism. All species undergo development of eggs in the mantle until the veliger stage, which is shed into the water, where development takes some weeks (Gallagher \& Reid, 1979; Reid, 1986). Subject to coastal water currents, etc. the effective population size may be large (Scheltema, 1986). Although they can live longer, there appears to be annual recruitment and turnover. This was demonstrated by Gallagher \& Reid (1979) for $L$. angulifera and would account for the seasonal differences in average size observed in $L$. pallescens (this study).

There are two possible explanations for the difference between species which live on bark and those which live on leaves. As in other examples of background-matching in a wide variety of animals, the tendency of the bark-living species to match their backgrounds must be the result of selection for crypsis. Polymorphism in the leaf-living species then arises either (i) because there is balancing selection maintaining the polymorphism, or (ii) because their location removes the individuals from predators, so that distinct mutants are not eliminated. There is then a balance between mutation and random loss. If selection on the colour morphs is demonstrated sufficiently often then the neutral hypothesis cannot be sustained. Various observations and experiments have shown that selection may act in these species to affect morph frequency (Cook, 1990; Hughes \& Mather, 1986; Reid, 1987). Experiments to test for selection sometimes fail to demonstrate it (Reid, 1987; Cook \& Garbett, 1992). It is possible to consider the alternative hypothesis, that for the most part the forms are neutral and that the species on leaves are polymorphic due to neutral mutations, despite the fact that selection sometimes intervenes. The question is, can the neutral model be applied to the data in such away as to support or reject this hypothesis?

\section{Application of theory}

If the forms are neutral and there are other neutral loci which can be examined then a class of patterns exists which can be compared with expectations based on effective population size and mutation rate. Investigation of different systems in the same samples should provide similar estimates of inbreeding and effective population size. The modern theory starts from the infinite allele model. Unless genes are individually sequenced, however, the investigator is always dealing with phenotypes, each of which may be produced by numerous different base sequences. This is true whether the phenotype is a shell colour morph or an enzyme allele.

There is variation in the appearance of dark individuals, which almost certainly indicates the existence of genetic differences. If the phenotype is simply classified into dark and non-dark (equivalent to banded and unbanded), the theory can be examined on the basis of two forms with forward and back mutation. For alleles at frequencies $p$ and $q$ with mutation and back muta-

Table 1 Estimates of mean frequencies of dark and $4 N_{\mathrm{e}}(v+w)$ for samples from Papua New Guinea (Morobe and Salamaua), Thailand (Phuket Rhizophora and Avicennia samples) and Kenya. Dark individuals have predominantly olive brown shells, whereas non-darks are predominantly uniform yellow or orange (illustrated in Cook \& Garbett, 1989), but there is some variation within each phenotype category. Each phenotype may therefore include genetically distinct forms

\begin{tabular}{|c|c|c|c|c|c|c|c|c|}
\hline \multirow[b]{2}{*}{ Location } & \multirow[b]{2}{*}{ Number of samples } & \multirow[b]{2}{*}{ Sample size } & \multirow[b]{2}{*}{ Mean dark } & \multirow[b]{2}{*}{ Variance } & \multicolumn{2}{|c|}{$4 N_{\mathrm{e}}(v+w)$} & \multicolumn{2}{|l|}{$F_{\text {st }}$} \\
\hline & & & & & dom & rec & dom & rec \\
\hline Morobe & 58 & 90.2 & 0.705 & 0.00814 & 29.5 & 15.5 & 0.0328 & 0.0606 \\
\hline Salamaua & 10 & 63.6 & 0.675 & 0.00569 & 49.2 & 30.2 & 0.0200 & 0.0321 \\
\hline Phuket Rh & 31 & 70.9 & 0.838 & 0.00775 & 30.9 & 8.9 & 0.0323 & 0.1007 \\
\hline Phuket Av & 19 & 56.3 & 0.716 & 0.02018 & 10.4 & 7.0 & 0.0876 & 0.1549 \\
\hline Kenya & 8 & 48.9 & 0.560 & 0.00729 & 29.6 & 24.9 & 0.0326 & 0.0387 \\
\hline Weighted mean & & 75.6 & 0.728 & 0.00953 & 25.2 & 12.2 & 0.0382 & 0.0760 \\
\hline
\end{tabular}


tion rates of $v$ and $w$, the equilibrium frequency is $q=v /(v+w)$. The variance is (Gale, 1990),

$V=v w /\left\{\left[1+4 N_{\mathrm{e}}(v+w)\right](v+w)^{2}\right\}$

where $N_{\mathrm{e}}$ is the effective population size. Consequently, $4 N_{\mathrm{e}}(v+w)=p q / V-1$.

Similarly, for a pair of alleles the inbreeding coefficient $F_{\text {ST }}$ is $V / p q$ (Lewontin \& Krakauer, 1975; Kimura, $1983)$, so that $4 N_{\mathrm{e}}(v+w)=(1-F) / F$.

The observed sample variances have been calculated for five sets of homogeneous samples of Littoraria pallescens from three areas [Papua New Guinea (Cook, 1986), Thailand (Cook, 1990) and Kenya (L. M. Cook, unpublished)]. Each set of samples comes from several kilometres of coastline. Estimates of $4 N_{\mathrm{e}}(v+w)$ are calculated in Table 1, assuming first that dark is dominant, and second that it is recessive. Both dominant and recessive banding alleles are known in other mollusc species (Murray, 1975), but dominance is more probable. Frequencies of dark are similar in all parts of the range, as are frequencies of orange compared with yellow, and the average values of $4 N_{\mathrm{e}}(v+w)$ obtained are, respectively, 25.2 and 12.2. Estimates of $F$ have also been included in Table 1 because of the close relation between the two statistics and the use of $F$ in the Lewontin-Krakauer test. Small values for $N_{\mathrm{e}}(v+w)$ are those much less than unity. The implication is therefore that effective population size is large in this species.

The problem with accepting this conclusion at its face value is that we have no means of knowing the value of $v$ or $w$. These quantities are the conversion rates from one phenotype to the other. Observed rates for such phenotypes are of the order of $10^{-6}-10^{-5}$, giving a range of values of $N$ from $1.5 \times 10^{5}$ to $3.2 \times 10^{6}$. But what mutation rate should we use if, for example, we wish to use these data to predict the allele frequency distribution for a set of proteins studied by electrophoresis?

The conversion rate is a consequence of the mutation rate of each base, and generally quoted figures for that are $10^{-9}-10^{-8}$ (Kimura, 1986). One way of relating the two levels would be to assume that base mutations have probabilities in the ratio $v: w$ of bringing about conversion of the phenotype. If there are $c$ bases, each with a mutation rate $u$, then the probability that at least one mutation will occur is $1-(1-u)^{c}$, or $1-\mathrm{e}^{-c u}$. This is approximately equal to $c u$, provided $c u$ is small, and is the frequency of detectable mutations of one kind or the other. Consequently, $c u=v+w$. The phenotype forward and back mutation rates would then be the product of this value and frequencies $v /(v+w)$ or $w /(v+w)$.
The same result is obtained by starting with $c$ alleles, each of which can mutate to any other, while only a fraction of these cause phenotypic conversion. The change in gene frequency due to mutation for two alleles, distinguished by subscripts, is

$\Delta q_{1}=-u_{21} q_{1}+u_{12} q_{2}$,

$\Delta q_{2}=u_{21} q_{1}-u_{12} q_{2}$.

In the previous notation, $u_{21}=v$ and $u_{12}=w$. At equilibrium, there is no change in gene frequency, so that

$\left[\begin{array}{cc}-u_{21} & u_{12} \\ u_{21} & -u_{12}\end{array}\right]\left[\begin{array}{l}q_{1} \\ q_{2}\end{array}\right]=\left[\begin{array}{ll}\frac{\delta \Delta q_{1}}{\delta q_{1}} & \frac{\delta \Delta q_{1}}{\delta q_{2}} \\ \frac{\delta \Delta q_{2}}{\delta q_{1}} & \frac{\delta \Delta q_{2}}{\delta q_{2}}\end{array}\right]\left[\begin{array}{l}q_{1} \\ q_{2}\end{array}\right]=0$.

Similarly, for $c$ alleles,

$\Delta Q \cdot Q=0$.

The two terms represent the matrix of partial derivatives and the vector of frequencies, respectively, with dimensions $c \times c$ and $c$. Suppose that $a$ of these alleles have one phenotype and $c-a$ of them have the other. The frequencies of the two phenotypes at equilibrium are,

$A=\sum_{i=1}^{a} q_{i}\left(1-\sum_{j=a+1}^{c} u_{i j}\right)+\sum_{i=a+1}^{c} q_{i} \sum_{j=1}^{a} u_{j i}$,

and

$1-A=\sum_{i=a+1}^{c} q_{i}\left(1-\sum_{j=1}^{a} u_{j i}\right)+\sum_{i=1}^{a} q_{i} \sum_{j=a+1}^{c} u_{j i}$.

If all the $u_{i j}$ have the same value, then the two phenotypes have frequencies

$A=\sum_{1}^{a} q_{i}[1-(c-a) u]+\sum_{a+1}^{c} q_{i} a u$,

and

$1-A=\sum_{a+1}^{c} q_{i}(1-a u)+\sum_{1}^{a} q_{i}(c-a) u$.

The phenotypic conversion rates are therefore $(c-a) u$ and $a u$ respectively, equal to $v$ and $w$ of the first notation. The equilibrium frequency is $a / c$ and $v+w=c u$. The average number of bases per gene is $10^{3}$ or more, so that with $u=10^{-9}$ or higher the implication is that the conversion rate should be $10^{-6}$ or higher. 


\section{Comparison of datasets}

If we accept the calculation for the colour morphs as indicating that the effective population size is larger than average, then for effectively neutral systems in which several alleles representing several base mutations are detectable, we should see strongly L-shaped frequency distributions. This would therefore be the prediction for a survey of sequenced genes, if the majority of the loci are neutral. The number of alleles present is also predicted in theory, and is in principle a more reliable statistic (Ewens, 1979a). On the other hand, if the colour morph frequency is constant because the background is constant and selection on it leads to a constant equilibrium, then other loci might show a quite different pattern. The effective population size estimated from colour frequency may be larger than that indicated by other loci.

The only data so far available for mangrove Littoraria are for the Caribbean species $L$. angulifera. Gaines et al. (1974) provide frequencies for an esterase locus in 20 populations from islands in the Gulf of Mexico, Florida, while Janson (1985) examined 12 enzyme loci in the same region and the Atlantic coast of Florida, five of which were polymorphic. Although not a leaf species, $L$. angulifera varies in shell colour, with marked differences recorded between populations (Janson, 1985).

Much work had been published on expected frequency distributions of alleles and of heterozygosity in neutral multiallelic systems (e.g. by Ewens, 1972, 1979a, b; Ewens \& Li, 1980; Watterson, 1978) but difficulty is experienced in using the theory to interpret real data (e.g. Mani et al., 1986). In the present case, each of these systems has therefore been treated as a morph pair, to allow direct comparison with the colour data (this was the approach adopted by Wright in developing his theory in relation to multiple alleles). Alleles have been grouped into two electrophoretic mobility categories (phenotypes), depending on whether they are more or less mobile than a central value. The procedure is not quite as rash as it sounds because the drift dynamics remain binomial for arbitrary groupings of alleles into two categories. Also, the products of the mean frequencies and the empirical variances by which they are divided both vary in the same way, an extreme frequency producing a small product and tending to result in a small variance. The results are shown in Table 2.

The enzyme data give estimates for $N_{\mathrm{e}}(v+w)$ as large as or larger than those for colour in L. pallescens. The implication is either that all loci studied are subject to selection for constancy of phenotype frequency, which is unlikely, or that large effective population size does indeed stabilize phenotype frequencies in these species.

All estimates of variance are made directly from the estimated frequencies in each sample. This variance is composed of a component due to sampling by the investigator and a component due to true fluctuation from site to site. When the first component is estimated as the binomial variance, obtained from mean frequency and mean sample size, it often equals or exceeds the calculated variances of Tables 1 and 2 . These are therefore minimum estimates of effective population size, which, taken at face value, is sometimes virtually infinite.

It should be noted that the geographical distances involved are far greater for the $L$. pallescens data than for the $L$. angulifera data; thousands of kilometres between the groups in $L$. pallescens, hundreds for the Janson data and tens of kilometres between samples

Table 2 Data for enzyme loci and shell colour in L. angulifera analysed as if only two morphs are detectable for each locus, as in Table 1. For each system, allele frequencies have been pooled depending on whether the alleles are more or less mobile than a central value. Data from Gaines et al. (1974) and Janson (1985)

\begin{tabular}{|c|c|c|c|c|c|c|}
\hline System & $\begin{array}{l}\text { Number of } \\
\text { samples }\end{array}$ & $\begin{array}{l}\text { Sample } \\
\text { size }\end{array}$ & Mean & Variance & $4 N_{\mathrm{e}}(v+w)$ & $F_{\mathrm{ST}}$ \\
\hline \multicolumn{7}{|c|}{ Gaines et al. $(1974)$} \\
\hline Est & 20 & 65.6 & 0.818 & 0.0029 & 64.3 & 0.0153 \\
\hline \multicolumn{7}{|c|}{ Janson (1985) } \\
\hline Pgm & 4 & 34.0 & 0.510 & 0.0044 & 55.8 & 0.0176 \\
\hline Lap & 4 & 30.5 & 0.861 & 0.0080 & 14.0 & 0.0668 \\
\hline Ap & 4 & 30.8 & 0.766 & 0.0095 & 17.9 & 0.0530 \\
\hline Mpi & 4 & 33.8 & 0.662 & 0.0065 & 33.4 & 0.0290 \\
\hline Pgi & 4 & 34.3 & 0.440 & 0.0014 & 175.0 & 0.0056 \\
\hline Colour & 4 & 23.3 & 0.460 & 0.1342 & 0.85 & 0.5403 \\
\hline
\end{tabular}


for the data of Gaines et al (1974). In addition, the one exceptional system concerns colour in $L$. angulifera (Table 2). Here, there was great variation between the four sites, one sample consisting entirely of white shells whereas this category was lacking elsewhere. Evidently, the situation is quite different from that in L. pallescens or in bark-living Indo-Pacific species such as $L$. intermedia, which do not show such radical changes from place to place.

\section{Conclusion}

One way to examine the applicability of the neutral model as an explanation for the constancy of morph frequency in $L$. pallescens is to estimate the effective population size and compare the estimate with those for other genetic systems. The large estimate obtained for the visible morphs could be a good one, or an artifact arising because of stabilizing selection on morph frequency. If it was equally large in the majority of systems studied the neutral mutation theory would be strengthened as an acceptable explanation for the observed pattern.

In making the comparison there is a problem of difference in the level of detectability of alleles when different categories of the genetic system are studied. The rate of mutation detected is a product of the base mutation rate, the number of bases available to mutate and the probability that the mutation will result in an observable phenotypic change. Ewens (1972) pointed out that the number of allelic types and the sample size provide the information needed to generate the allelic frequency distribution and estimate the statistics related to $N_{\mathrm{e}}$ and the mutation rate. Because of the uncertainty of detection, however, we cannot determine how many alleles are present, whether we are dealing with allozymes or colour morphs. It may be possible to use the standard methods for comparisons between different data belonging to the same category of systems (allozymes, for example), but not between categories. Here, all systems have been treated as being composed of two morphs, like the visible polymorphism. Although information is lost in the process the mutation rates become comparable, allowing comparable estimates of $N$ to be made. Unless this is done, estimates of products of $N$ and mutation rate cannot be compared, because the implied mutation rate differs from system to system by orders of magnitude, depending on the detectability of allele differences.

Applying the method to enzyme data for the New World bark-living $L$. angulifera leads to estimates comparable to, or larger than, those for colour in $L$. pallascens. Recent work by Johnson \& Black (1991), on a littorinid species with benthic egg masses, shows much higher $F$ values, indicating small $N_{\mathrm{e}}$. Perhaps the effective population size in Littoraria is indeed exceptionally large. Therefore, mutation and diffusion may be responsible for the polymorphism and the constancy of morph frequency in this species, over a geographical range extending from east Africa to Papua New Guinea, with occasional perturbations caused by local selective events. If we consider the colour system alone, the only way this hypothesis could be rejected would be to accumulate sufficient observations as to make the evidence for balancing selection overwhelming. Estimates from a range of enzyme loci should also be made for $L$. pallescens, for direct comparison with the shell colour system. This could be done using different populations for estimates from different loci, in order to increase the independence of the estimates. It would then be possible to say whether the alternative types of data produce consistent or inconsistent indicators of effective population size.

\section{Acknowledgements}

Material was collected with support from the Nuffield Foundation (Kenya), the Royal Society (Papua New Guinea), the Linnean Society and British Ecological Society (Thailand). I am grateful to G. S. Mani for discussion of this problem and to J. R. G. Turner for the suggestion that the best fate for unfunded grant proposals is to be published.

\section{References}

CAIN, A. J. 1983. Ecology and ecogenetics of terrestrial molluscan populations. In: Russell-Hunter, W. D. (ed.) The Mollusca. Vol. 6. Ecology. Academic Press, London. pp. 597-647.

CAMERon, R. A. D. AND DILlon, P. J. 1984. Habitat, stability, population histories and patterns of variation of Cepaea nemoralis. Malacologia, 25, 271-290.

CLARKE, B., ARTHUR, W., HORSLEY, D. T. AND PARKIN, D. T. 1978. Genetic variation and natural selection in pulmonate molluscs. In: Fretter, V. and Peake, J. (ed.) Pulmonates. Vol. 2A, Systematics, Evolution and Ecology. Academic Press, New York. pp. 219-270.

CoOK, L. M. 1986. Site selection in a polymorphic mangrove snail. Biol. J. Linn. Soc., 29, 101-113.

CooK, L. M. 1990. Systematic effects on morph frequency in the polymorphic mangrove snail Littoraria pallescens. Heredity, 65, 423-427.

COOK, L. M. AND GARBETT, S. D. 1989. Patterns of variation in mangrove littorinid molluscs on Phuket island. Phuket Mar. Biol. Cent. Res. Bull., 53, 1-14.

COOK, L. M. AND GARBETT, S. D. 1992. Selection in the polymorphic mangrove snail Littoraria pallescens. In: Grahame, J., Mill, P. J. and Reid, D. G. (eds) Proceedings of 
the Third International Symposium on Littorinid Biology. Melacological Society, London (in press).

EWENS, w. J. 1982. The sampling theory of selectively neutral alleles. Theor. Pop. Biol., 3, 87-112.

EWENS, w. J. 1979a. Testing the generalized neutrality hypothesis. Theor. Pop. Biol., 15, 205-216.

EWEns, w. J. 1979b. Mathematical Population Genetics. Springer-Verlag, Berlin.

EWENS, W. J. AND L1, W.-H. 1980. Frequency spectra of neutral and deleterious alleles in a finite population. J. Math. Biol., 10, $155-166$.

GAINES, M. S., CALDWELL, J. AND VIVAS, A. M. 1974. Genetic variation in the mangrove periwinkle Littorina angulifera. Mar. Biol., 27, 327-332.

gale, J. s. 1990. Theoretical Population Genetics. Unwin Hyman, London. 417p.

Gallagher, S. B. AND REID, G. K. 1979. Population dynamics and zonation in the periwinkle snail, Littorina angulifera, of the Tampa Bay, Florida region. Nautilus, 94, 162-178.

HUGHES, J. M. AND MATHER, P. B. 1986. Evidence for predation as a factor in determining shell colour frequencies in a mangrove snail Littorina sp. (Prosobranchia: Littorinidae). Evolution, 40, 68-77.

JANSON, K. 1985. Genetic and morphologic variation within and between populations of Littorina angulifera from Florida. Ophelia, 24, 125-134.

JOHNSON, M. S. AND BLACK, R. 1991. Genetic subdivision of the intertidal snail Bembicium vittatum (Gastropoda: Littorinidae) varies with habitat in the Houtman Abrolhos Islands, Western Australia. Heredity, 67, 205-213.

JONES, J. S., LEITH, B. H. AND RAWLINGS, P. 1977. Polymorphisms in Cepaea: a problem, with too many solutions? Ann. Rev. Ecol. Syst., 8, 109-143.
K1MURA, M. 1983. The Neutral Theory of Molecular Evolution. Cambridge University Press, Cambridge.

K1MUrA, M. 1986. DNA and the neutral theory. Phil. Trans. Roy. Soc. Lond. B, 312, 343-354.

LEWONTIN, R. C. AND KRAKAUER, J. 1973. Distribution of gene frequency as a test of the theory of the selective neutrality of polymorphisms. Genetics, 74, 175-195.

MAN1, G. S., COOK. L. M. AND MARVDASHT1, R. 1986. What can be learnt about selection from gene frequency distribution? Genetics, 114, 971-982.

MURRAY, J. 1975. The genetics of the Mollusca. In: King, R. C. (ed.) Handbook of Genetics. Vol. 3. Invertebrates of Genetic Interest. New York, Plenum, pp. 3-31.

oCHMAN, H., JONES, J. S. AND SElANDER, R. K. 1983. Molecular area effects in Cepaea. Proc. Natl. Acad. Sci., U.S.A., 80, 4189-4193.

REID, D. G. 1986. The Littorinid Molluscs of Mangrove Forests in the Indo-Pacific Region: the Genus Littoraria. British Museum (Natural History), London.

RE1D, D. G. 1987. Natural selection for apostasy and crypsis acting on the shell colour polymorphism of a mangrove snail, Littoraria filosa (Sowerby) (Gastropoda: Littorinidae). Biol. J. Linn. Soc., 30, 1-24.

SCHELTEMA, R. S. 1986. On dispersal and planktonic larvae of benthic invertebrates: an eclectic overview and summary of the problem. Bull. Mar. Sci., 39, 290-322.

SELANDER, R. K. AND OCHMAN, H. 1983. The genetic structure of populations as illustrated by molluscs. In: Isozymes: Current Topics in Biological and Medical Research. Vol. 10: Genetics and Evolution. A. Liss, New York, pp. 93-123.

WATTERSON, G. A. 1978. The homozygosity test of neutrality. Genetics, 88, 405-417. 